\title{
Erlotinib is effective in pancreatic cancer with epidermal growth factor receptor mutations: a randomized, open-label, prospective trial
}

\author{
Jack P. Wang ${ }^{1,2,3, *}$, Chen-Yi Wu ${ }^{4,5,6, *}$, Yi-Cheng Yeh ${ }^{2,7}$, Yi-Ming Shyr ${ }^{2,8}$, Ying-Ying \\ $\mathbf{W u}^{1,2}$, Chen-Yu Kuo ${ }^{1,2,9,10}$, Yi-Ping Hung ${ }^{1,2,4,10}$, Ming-Huang Chen ${ }^{2,10}$, Wei-Ping \\ Lee $^{2,11}$, Jiing-Chyuan Luo ${ }^{1,2}$, Yee Chao ${ }^{1,2,12}$ and Chung-Pin Li ${ }^{1,2}$ \\ ${ }^{1}$ Division of Gastroenterology, Department of Medicine, Taipei Veterans General Hospital, Taipei, Taiwan \\ ${ }^{2}$ National Yang-Ming University School of Medicine, Taipei, Taiwan \\ ${ }^{3}$ Division of Gastroenterology, Department of Internal Medicine, Renai Branch, Taipei City Hospital, Taipei, Taiwan \\ ${ }^{4}$ Institute of Public Health, National Yang-Ming University, Taipei, Taiwan \\ ${ }^{5}$ Department of Dermatology, Taipei Veterans General Hospital, Taipei, Taiwan \\ ${ }^{6}$ Department of Dermatology, Heping Fuyou Branch, Taipei City Hospital, Taipei, Taiwan \\ 7 Department of Pathology, Taipei Veterans General Hospital, Taipei, Taiwan \\ ${ }^{8}$ Division of General Surgery, Department of Surgery, Taipei Veterans General Hospital, Taipei, Taiwan \\ ${ }^{9}$ Department of Medicine, National Yang-Ming University Hospital, Yilan, Taiwan \\ ${ }^{10}$ Division of Oncology, Department of Medicine, Taipei Veterans General Hospital, Taipei, Taiwan \\ ${ }^{11}$ Department of Medical Research, Taipei Veterans General Hospital, Taipei, Taiwan \\ 12 Department of Oncology Medicine, Taipei Veterans General Hospital, Taipei, Taiwan \\ * These authors contributed equally to this manuscript \\ Correspondence to: Chung-Pin Li, email: cpli@vghtpe.gov.tw \\ Keywords: adenocarcinoma; pancreas; erlotinib; epidermal growth factor receptor \\ Received: November 18, $2014 \quad$ Accepted: May 12, $2015 \quad$ Published: May 20, 2015
}

This is an open-access article distributed under the terms of the Creative Commons Attribution License, which permits unrestricted use, distribution, and reproduction in any medium, provided the original author and source are credited.

\section{ABSTRACT}

Objective To analyze the efficacy of gemcitabine with or without erlotinib for pancreatic cancer, and to determine the predictive role of epidermal growth factor receptor (EGFR) and KRAS mutations in these patients.

Methods This was a single-center, randomized, open-label, prospective trial. Eighty-eight chemotherapy-naïve metastatic pancreatic cancer patients were randomized for treatment with gemcitabine or gemcitabine plus erlotinib. EGFR and KRAS mutations were analyzed, respectively. The primary endpoint was the disease control rate.

Results Disease control rate (64\% vs. $25 \%$; $P<0.001)$, progression-free survival (median 3.8 vs. 2.4 months; $P<0.001$ ), and overall survival (median 7.2 vs. 4.4 months; $P<0.001$ ) were better in the gemcitabine plus erlotinib group than in the gemcitabine alone group. In the gemcitabine plus erlotinib group, disease control (85\% vs. 33\%; $P=0.001$ ), progression-free survival (median 5.9 vs. 2.4 months; $P$ $=0.004$ ), and overall survival (median 8.7 vs. 6.0 months; $P=0.044$ ) were better in patients with EGFR mutations than in those without EGFR mutations. KRAS mutation was not associated with treatment response or survival.

Conclusions Gemcitabine plus erlotinib is more effective than gemcitabine alone for treating metastatic pancreatic cancer patients, especially those with EGFR mutations. ClinicalTrials.gov number, NCT01608841. 


\section{INTRODUCTION}

Pancreatic cancer is a rapidly progressive malignancy with a 5-year survival rate of $<10 \%$ [1]. Gemcitabine is the standard chemotherapy for metastatic pancreatic adenocarcinoma, but shows an objective response rate of only $5-10 \%$ and a median survival of 5.76.8 months [2-5]. During the last decade, many trials have been conducted on combined treatment with gemcitabine and targeted agents in an attempt to improve treatment outcome [6-8]. Unfortunately, most trials have failed to demonstrate any survival benefit compared to gemcitabine alone.

The mutation status of the EGFR tyrosine kinase (TK) domain is a predictive factor for EGFR inhibitor therapy in non-small cell lung cancer (NSCLC), especially among the Chinese people [9-14]. Whether this result applies to pancreatic cancer remains unclear. Erlotinib, a selective epidermal growth factor receptor (EGFR) tyrosine kinase inhibitor (TKI), is an orally active agent for advanced NSCLC and pancreatic cancer $[3,15]$. The phase III NCIC CTG PA.3 trial comparing gemcitabine plus erlotinib and gemcitabine alone in advanced pancreatic cancer is the only trial of a targeted agent in pancreatic cancer that has shown a statistically significant improvement in survival [3]. However, the survival was only slightly longer for the gemcitabine plus erlotinib arm versus the gemcitabine plus placebo arm (median $6.24 \mathrm{vs}$. 5.91 months, $P=0.038$ ). Considering cost effectiveness and the survival benefit, it is important to be able to accurately select the subgroup of patients who could benefit from this therapeutic regimen.
Pancreatic cancer shows the highest frequency of $K R A S$ gene mutations among human cancers [16]. Ras signaling pathways are commonly activated in tumors and are involved in mediating the downstream effects of EGFR activation. Greater than $95 \%$ of these mutations involve codon 12 or 13 (exon 2), and a few involve codon 61 (exon 3) [16]. Whether KRAS mutations are associated with less efficient $E G F R$-directed targeted therapy in pancreatic cancer patients remains controversial and requires further investigation $[17,18]$.

To clarify the effects of adding erlotinib to gemcitabine and the roles of EGFR and KRAS mutations as predictive biomarkers in patients with advanced pancreatic cancer who receive these regimens, we performed this open-label, randomized, prospective study.

\section{RESULTS}

\section{Patient characteristics}

Between July 2005 and June 2012, 88 patients were randomly assigned (44 gemcitabine plus erlotinib and 44 gemcitabine alone) (Figure 1). Baseline characteristics (Table 1) were well matched between the gemcitabine plus erlotinib arm and the gemcitabine alone arm. The median follow-up time was 7.2 months (range, 0.1-30.5 months) for the gemcitabine plus erlotinib group and 4.5 months (range, 0.4-16.9 months) for the gemcitabine alone group.

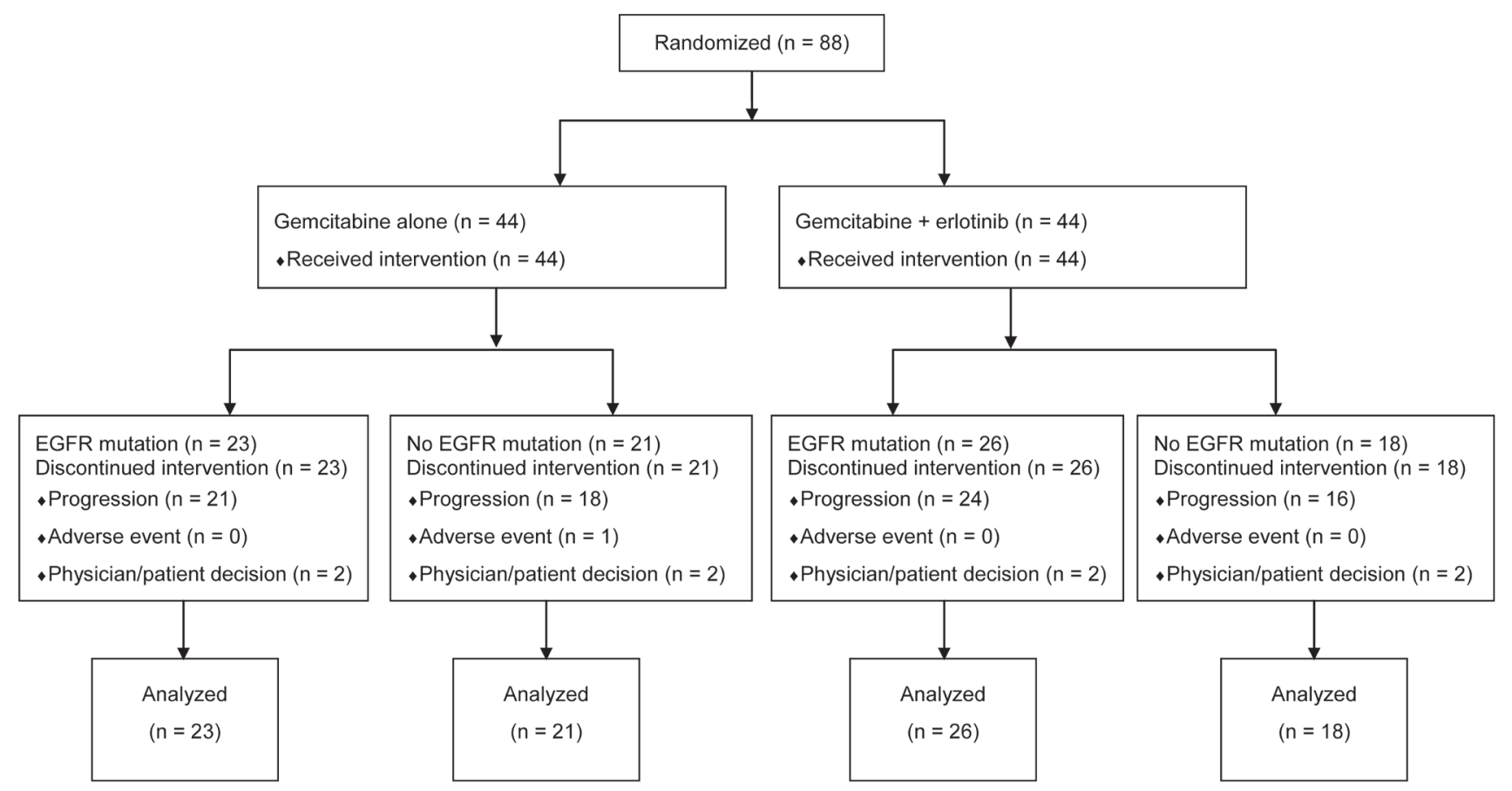

Figure 1: CONSORT diagram. 
Table 1: Patient characteristics

\begin{tabular}{|c|c|c|c|c|c|c|}
\hline \multirow[b]{2}{*}{ Characteristics } & \multirow{2}{*}{$\begin{array}{l}\text { All patients } \\
\qquad(\mathrm{n}=88)\end{array}$} & \multicolumn{2}{|c|}{ Gemcitabine alone $(n=44)$} & \multicolumn{2}{|c|}{ Gemcitabine + erlotinib $(\mathrm{n}=44)$} & \multirow[b]{2}{*}{$P$} \\
\hline & & $\begin{array}{c}\text { EGFR Mutation }(+) \\
(\mathrm{n}=23)\end{array}$ & $\begin{array}{c}\text { EGFR Mutation (-) } \\
\qquad(\mathrm{n}=21)\end{array}$ & $\begin{array}{c}E G F R \text { Mutation }(+) \\
\qquad(\mathrm{n}=26)\end{array}$ & $\begin{array}{c}\text { EGFR Mutation (-) } \\
\qquad(\mathrm{n}=18)\end{array}$ & \\
\hline Gender & & & & & & 0.711 \\
\hline Male & $65(74 \%)$ & $16(70 \%)$ & $17(81 \%)$ & $20(77 \%)$ & $12(67 \%)$ & \\
\hline Female & $23(26 \%)$ & $7(30 \%)$ & $4(19 \%)$ & $6(23 \%)$ & $6(33 \%)$ & \\
\hline Age (Years) & & & & & & 0.160 \\
\hline Median & 70 & 67 & 74 & 69 & 65 & \\
\hline Range & $33-91$ & $37-86$ & $33-91$ & $35-86$ & $43-84$ & \\
\hline Performance status & & & & & & 0.057 \\
\hline 0 & $73(83 \%)$ & $20(87 \%)$ & $16(76 \%)$ & $21(81 \%)$ & $16(89 \%)$ & \\
\hline 1 & $13(15 \%)$ & $3(13 \%)$ & $5(24 \%)$ & $5(19 \%)$ & $0(0 \%)$ & \\
\hline 2 & $2(2 \%)$ & $0(0 \%)$ & $0(0 \%)$ & $0(0 \%)$ & $2(11 \%)$ & \\
\hline Relapse after surgery & $20(23 \%)$ & $4(17 \%)$ & $7(33 \%)$ & $5(19 \%)$ & $4(22 \%)$ & 0.593 \\
\hline \multicolumn{7}{|l|}{ Metastatic site } \\
\hline Liver & $61(69 \%)$ & $19(83 \%)$ & $14(67 \%)$ & $17(65 \%)$ & $11(61 \%)$ & 0.434 \\
\hline Peritoneum & $49(56 \%)$ & $15(65 \%)$ & $10(48 \%)$ & $17(65 \%)$ & $7(39 \%)$ & 0.217 \\
\hline Lung & $18(20 \%)$ & $2(9 \%)$ & $5(24 \%)$ & $6(23 \%)$ & $5(28 \%)$ & 0.423 \\
\hline Adrenal gland & $1(1 \%)$ & $1(4 \%)$ & $0(0 \%)$ & $0(0 \%)$ & $0(0 \%)$ & 0.414 \\
\hline Bone & $2(2 \%)$ & $0(0 \%)$ & $1(5 \%)$ & $1(4 \%)$ & $0(0 \%)$ & 0.609 \\
\hline Others & $9(10 \%)$ & $3(13 \%)$ & $2(10 \%)$ & $2(8 \%)$ & $2(11 \%)$ & 0.939 \\
\hline Differentiation & & & & & & 0.479 \\
\hline Well & $2(2 \%)$ & $0(0 \%)$ & $0(0 \%)$ & $1(4 \%)$ & $1(6 \%)$ & \\
\hline Moderately & $18(20 \%)$ & $6(26 \%)$ & $6(29 \%)$ & $2(8 \%)$ & $4(22 \%)$ & \\
\hline Poorly & $13(15 \%)$ & $4(17 \%)$ & $4(19 \%)$ & $2(8 \%)$ & $3(17 \%)$ & \\
\hline Unknown & $55(63 \%)$ & $13(57 \%)$ & $11(52 \%)$ & $21(81 \%)$ & $10(56 \%)$ & \\
\hline \multicolumn{7}{|l|}{ CA19-9 (U/ml) } \\
\hline Elevated $(>37)$ & $71(81 \%)$ & $19(83 \%)$ & $19(90 \%)$ & $21(81 \%)$ & $12(67 \%)$ & 0.306 \\
\hline Median (range) in all & $1,083(2-1,439,400)$ & $1,169(4-10,411)$ & $725(14-97,622)$ & $1,072(8-439,380)$ & $1,048(2-1,439,400)$ & 0.397 \\
\hline patients & & & & & & \\
\hline
\end{tabular}

\section{Response and survival}

The disease control rate $(64 \%$ vs. $25 \% ; P<0.001)$, PFS (median 3.8 months; 95\% confidence interval [CI], 1.2 to 6.4 months vs. 2.4 months; $95 \%$ CI, 1.9 to 2.9 months; $P<0.001$ ), and OS (median 7.2 months; $95 \%$ CI, 5.3 to 9.0 months vs. 4.4 months; $95 \%$ CI, 3.0 to 5.9 months; $P<0.001)$ were significantly better in the gemcitabine plus erlotinib group than in the gemcitabine alone group (Table 2 and Figures 2A and 2B).

\section{EGFR mutation profile}

Forty-nine (56\%) patients had 62 mutations in EGFR exons 18-21 that caused substantial downstream amino acid changes (activating mutations, Supplementary Table 2). All mutations were heterozygous (Supplementary Figure 1) and confirmed by next-generation sequencing. 
Table 2: Evaluation of the effect of EGFR mutations on treatment response

\begin{tabular}{|c|c|c|c|c|c|c|}
\hline & \multicolumn{3}{|c|}{ Gemcitabine alone } & \multicolumn{3}{|c|}{ Gemcitabine + erlotinib } \\
\hline & $\begin{array}{c}\text { Mutation }(+) \\
n=23\end{array}$ & $\begin{array}{c}\text { Mutation (-) } \\
n=21\end{array}$ & $\mathrm{P}$ & $\begin{array}{c}\text { Mutation }(+) \\
n=26\end{array}$ & $\begin{array}{c}\text { Mutation (-) } \\
n=18\end{array}$ & $\mathrm{P}$ \\
\hline \multicolumn{7}{|l|}{ Objective response } \\
\hline Complete response & $0 / 23(0 \%)$ & $0 / 21(0 \%)$ & & $0 / 26(0 \%)$ & $0 / 18(0 \%)$ & \\
\hline Partial response & $1 / 23(4 \%)$ & $3 / 21(14 \%)$ & & $7 / 26(27 \%)$ & $1 / 18(6 \%)$ & \\
\hline Stable disease & $4 / 23(17 \%)$ & $3 / 21(14 \%)$ & & $15 / 26(58 \%)$ & $5 / 18(28 \%)$ & \\
\hline Progression of disease & $16 / 23(70 \%)$ & $12 / 21(57 \%)$ & & $2 / 26(8 \%)$ & $10 / 18(56 \%)$ & \\
\hline Not assessed* & $2 / 23(9 \%)$ & $3 / 21(14 \%)$ & & $2 / 26(8 \%)$ & $2 / 18(11 \%)$ & \\
\hline Response rate & $1 / 23(4 \%)$ & $3 / 21(14 \%)$ & 0.335 & $7 / 26(27 \%)$ & $1 / 18(6 \%)$ & 0.115 \\
\hline Disease control rate & $5 / 23(22 \%)$ & $6 / 21(29 \%)$ & 0.601 & $22 / 26(85 \%)$ & $6 / 18(33 \%)$ & 0.001 \\
\hline \multicolumn{7}{|l|}{ Best CA19-9 response } \\
\hline$>80 \%$ decline & $2 / 23(9 \%)$ & 4/21 (19\%) & 0.403 & $6 / 26(23 \%)$ & $2 / 18(11 \%)$ & 0.439 \\
\hline$>50 \%$ decline & $6 / 23(26 \%)$ & $6 / 21(29 \%)$ & 0.853 & $15 / 26(58 \%)$ & $4 / 18(22 \%)$ & 0.020 \\
\hline
\end{tabular}

Exon 20 was the most commonly mutated exon $(50 \%$ of the mutations), followed by exon 19 (37\%), exon 21 $(10 \%)$, and exon $18(3 \%)$. L778P in exon 20 was the most common mutation site (24\% of all mutations), followed by K728R (19\%) and W731X (13\%) in exon 19, and I821T $(15 \%)$ in exon 20. Patients with an L778P mutation who received gemcitabine plus erlotinib had a significantly higher disease control rate than those who received gemcitabine alone $(71 \%, N=7 v s .0 \%, N=8 ; P=0.007$, Supplementary Table 2).

Silent mutations were found in $18 / 88$ patients. Six patients had a Q787Q mutation in exon 20, and two of these had additional missense mutations. Four had an F795F mutation, and two of these had other missense mutations. Two had a D800D mutation together with other missense mutations. One had a Q812Q mutation in exon 20 with a simultaneous missense mutation (I821T). Five had a D830D mutation in exon 21, and of these, one had a D830D mutation only. In both the gemcitabine alone and the gemcitabine plus erlotinib groups, response rates, disease control rates, PFS, and OS were not different between patients with silent $E G F R$ mutations and patients without mutations. Patients with silent $E G F R$ mutations were counted as patients without $E G F R$ mutations in this study.

No activating EGFR mutation was found in the buffy coat of all patients, indicating that the mutations occurred somatically during carcinogenesis.

\section{EGFR mutations and tumor response}

In the gemcitabine alone group, response rate, disease control rate, and CA19-9 response rate were comparable between patients with and without EGFR mutations (Table 2). In the gemcitabine plus erlotinib group, patients with $E G F R$ mutations had a significantly higher disease control rate ( $85 \%$ vs. $33 \% ; P=0.001)$ and a significantly higher rate of a $>50 \%$ CA19-9 decline $(58 \%$ vs. $22 \% ; P=0.020)$.

\section{EGFR mutations and survival}

Patients receiving gemcitabine alone had similar PFS and OS regardless of the presence of EGFR mutations (Figures 3A and 4A). In the gemcitabine plus erlotinib group, patients with $E G F R$ mutations had a significantly longer PFS (5.9 months; 95\% CI, 4.5 to 7.3 months vs. 2.4 months; $95 \%$ CI, 1.5 to 3.3 months; $P=0.004$, Figure 3B) and a significantly longer OS ( 8.7 months; $95 \% \mathrm{CI}, 6.2$ to 11.1 months $v s .6 .0$ months; $95 \%$ CI, 3.0 to 8.9 months; $P$ $=0.044$, Figure 4B) than those without EGFR mutations. 


\section{$K R A S$ mutations and treatment response}

Tumor specimens from 83/88 pancreatic adenocarcinomas $(94.3 \%)$ had a point mutation of $K R A S$ in exon 2 or 3 . There were 74 mutations in exon 2 (codon 12 or 13), two mutations in exon 3 (codon 61), and seven mutations in both exon 2 and exon 3. KRAS mutations were not associated with clinical and pathologic parameters including age, sex, ECOG performance status, tumor location, histologic differentiation, levels of CA199, response to chemotherapy, PFS, and OS. Patients with $K R A S$ mutations did not have a statistically different rate of $E G F R$ mutations ( $58 \%$ vs. $20 \%, P=0.166$ ) compared to patients without KRAS mutations.

\section{Toxicity and dosage modifications}

Forty-four patients treated with gemcitabine and erlotinib and 44 treated with gemcitabine alone received at least one dose of study medication and were available
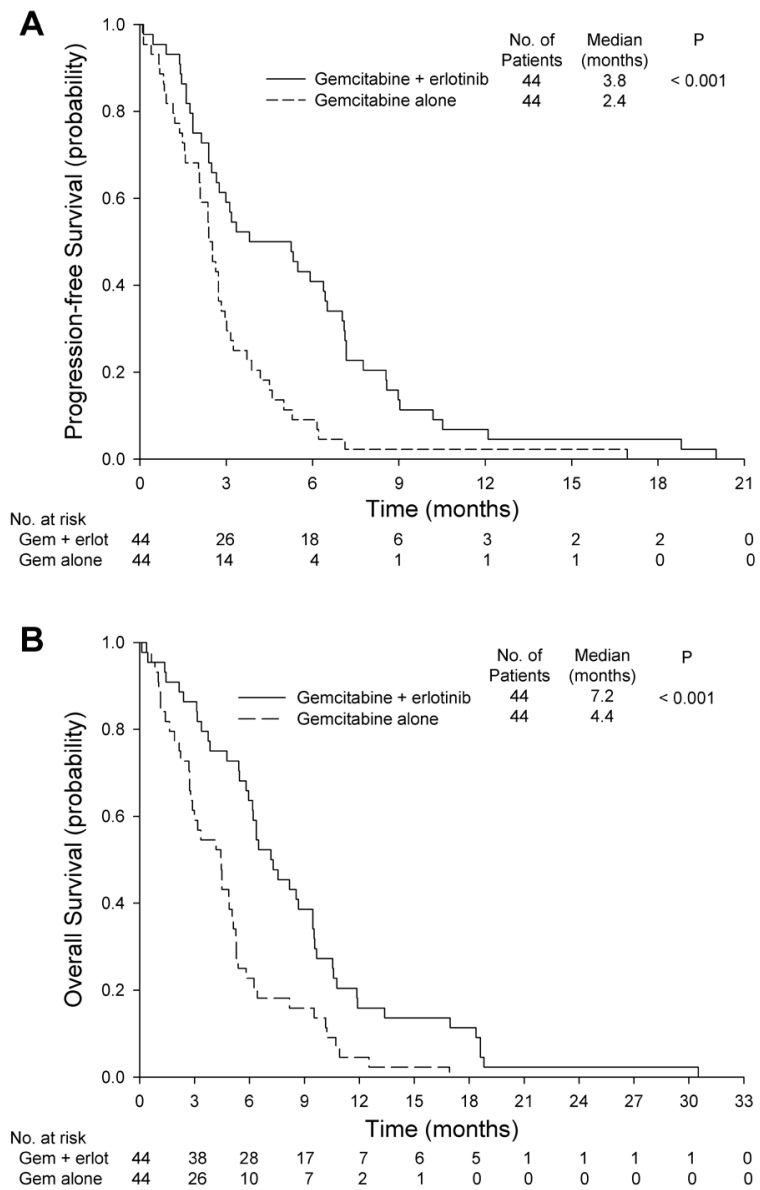

Figure 2: A. Progression-free survival for gemcitabine alone or gemcitabine plus erlotinib. B. Overall survival for gemcitabine alone or gemcitabine plus erlotinib. Gem: gemcitabine, Erlot: erlotinib. for assessment of toxicity. Adverse events are summarized in Table 3.

Treatment was generally well tolerated in both arms. Patients receiving gemcitabine plus erlotinib experienced a significantly higher frequency of rash $(64 \%$ vs. $2 \%$, $P<0.001)$, but these were generally grade 1 or 2 . The incidence of other adverse events, including hematologic toxicity and elevations in alanine aminotransferase, were similar between groups. There were no protocol-related deaths. One patient in the gemcitabine plus erlotinib group had interstitial lung disease (ILD)-like syndrome possibly related to therapy.

The gemcitabine dose intensity was similar in the gemcitabine alone and gemcitabine plus erlotinib groups

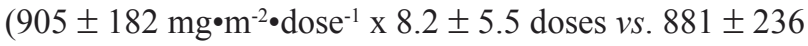
$\mathrm{mg} \cdot \mathrm{m}^{-2} \mathrm{dose}^{-1}$ x $17.1 \pm 13.4$ doses). Four (9\%) patients receiving erlotinib and gemcitabine had at least one dose reduction of their oral agent. The erlotinib dose intensity was $96.8 \pm 12.6 \mathrm{mg}^{\circ} \mathrm{m}^{-2}$ day $^{-1}$ x $17.9 \pm 13.7$ weeks.
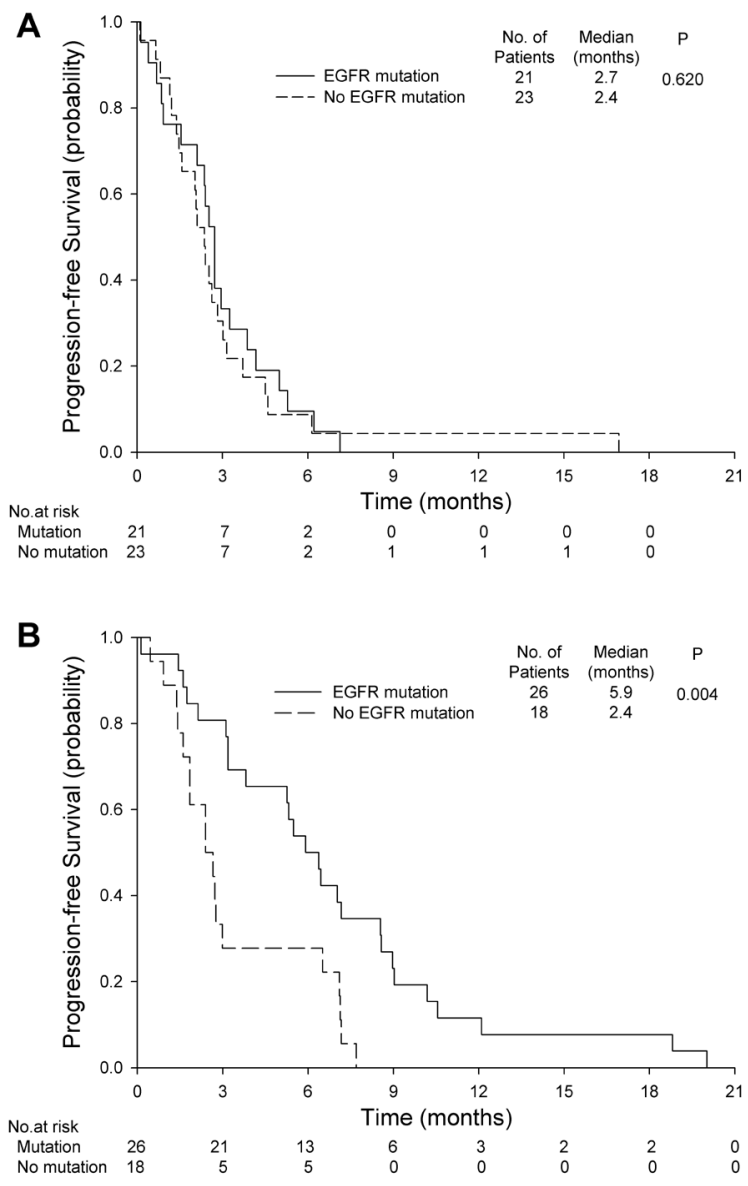

Figure 3: Progression-free survival for A. gemcitabine alone with or without $E G F R$ mutations and B. gemcitabine plus erlotinib with or without $E G F R$ mutations. 


\section{Skin rash and survival in the gemcitabine plus erlotinib group}

Of the 44 patients receiving gemcitabine plus erlotinib, 16 had no rash, 17 had grade $1 \mathrm{rash}, 7$ had grade 2 rash, 3 had grade 3 rash, and 1 had grade 4 rash. Patients with $E G F R$ mutations did not have a significantly different rate of skin rash than patients without EGFR mutations ( $73 \%$ vs. $50 \% ; P=0.118$ ). The presence of rash was associated with significantly higher rates of disease control $(75 \%$ vs. $44 \% ; P=0.038)$ and treatment response $(29 \%$ vs. $0 \% ; P=0.036)$. Patients with skin rash had significantly longer PFS (6.4 months; 95\% CI, 4.1 to 8.7 months vs. 2.4 months; $95 \%$ CI, 0.8 to 4.0 months; $P=0.006$, Figure $5 \mathrm{~A}$ ) and OS (8.6 months; $95 \%$ CI, 6.1 to 11.0 months vs. 5.8 months; $95 \%$ CI, 3.1 to 8.6 months; $P=0.043$, Figure 5B).

\section{DISCUSSION}

EGFR mutations are known to be the most important determinants of NSCLC patient response to EGFR-TKIs [9-14]. However, the importance of EGFR mutations in
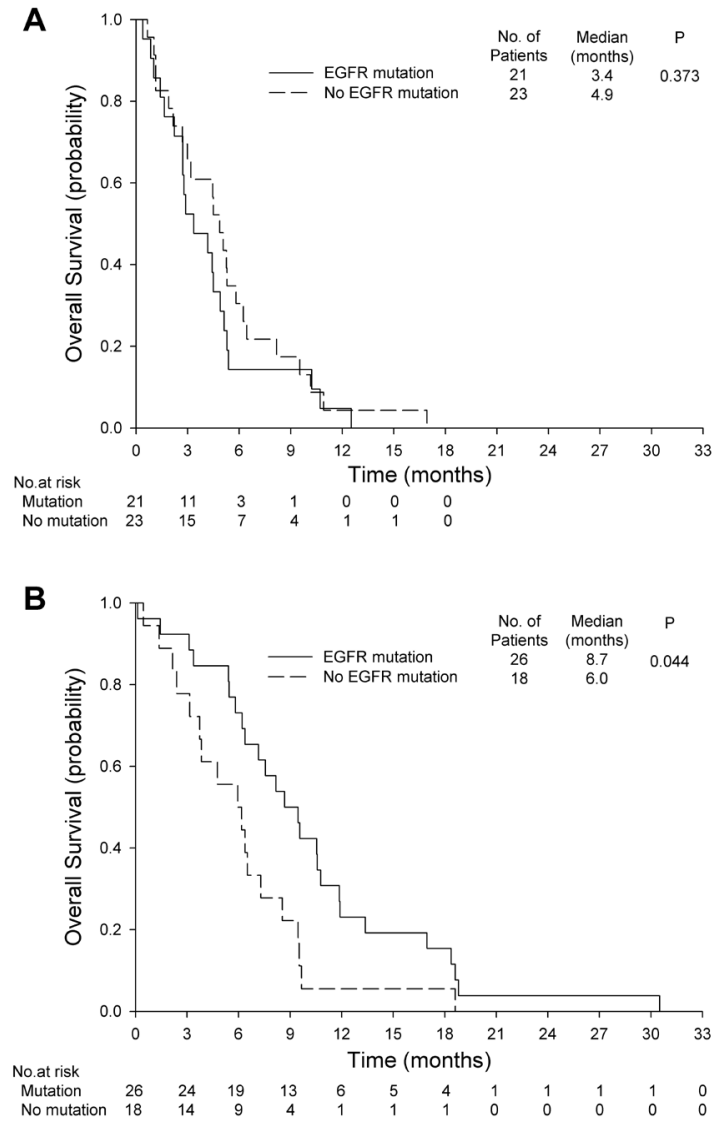

Figure 4: Overall survival for A. gemcitabine alone with or without $E G F R$ mutations and $\mathbf{B}$. gemcitabine plus erlotinib with or without $E G F R$ mutations. pancreatic cancer has not been investigated until now. The EGFR mutation profile in the Chinese population analyzed in this study can be used to predict treatment response and survival in pancreatic cancer patients receiving gemcitabine plus erlotinib.

The NCIC CTG PA.3 trial demonstrated the beneficial effects of erlotinib in the treatment of pancreatic cancer [3]. However, no Chinese patients were included in that study and the prolongation of median OS with erlotinib was only slightly longer, at 0.33 months. Neither EGFR protein expression status nor gene copy number was identified as a marker predictive of survival benefit following combination treatment with erlotinib and gemcitabine $[3,17]$. In the present study, we used direct sequencing to detect EGFR mutations and confirmed by next-generation sequencing, which accurately predicted treatment response and survival in Chinese pancreatic cancer patients.

The median OS of patients treated with gemcitabine plus erlotinib (regardless of EGFR mutations) was 7.2 months. The NCIC CTG PA.3 trial, which included both locally advanced pancreatic cancer and metastatic pancreatic cancer patients, showed that the median OS
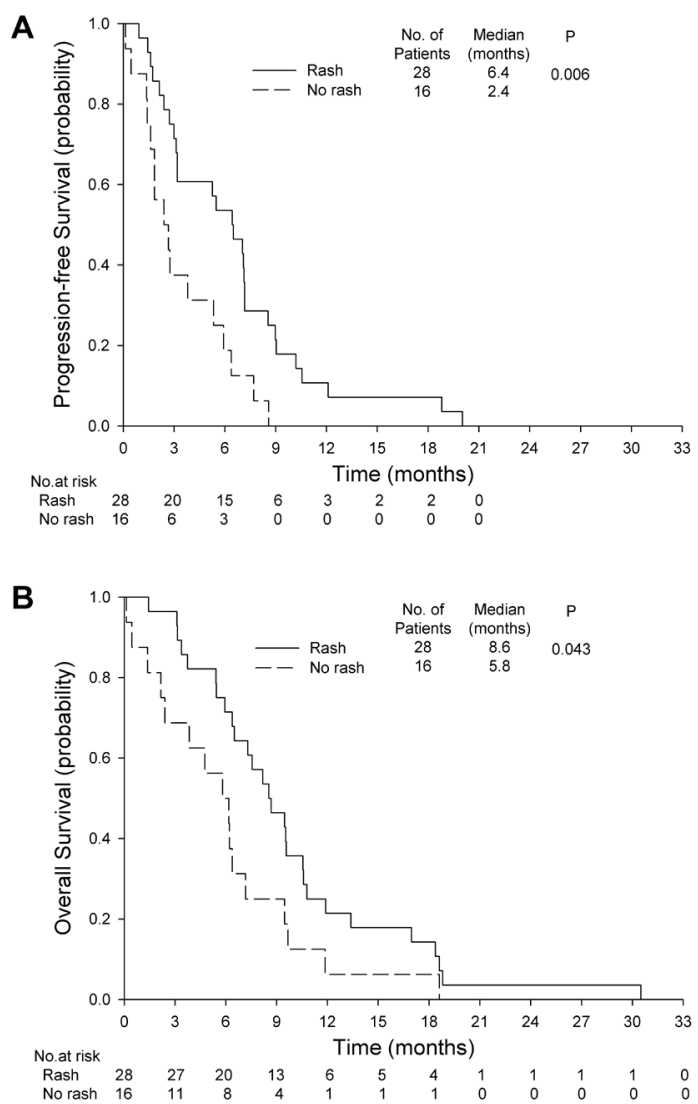

Figure 5: Skin rash and survival in the gemcitabine plus erlotinib group. A. progression-free survival B. overall survival. 


\begin{tabular}{|c|c|c|c|c|c|c|c|c|c|c|c|c|c|c|c|c|}
\hline \multirow[b]{3}{*}{ Grade } & \multicolumn{8}{|c|}{ Gemcitabine alone } & \multicolumn{8}{|c|}{ Gemcitabine + erlotinib } \\
\hline & \multicolumn{4}{|c|}{ Mutation $(+) n=23$} & \multicolumn{4}{|c|}{ Mutation (-) $n=21$} & \multicolumn{4}{|c|}{ Mutation $(+) n=26$} & \multicolumn{4}{|c|}{ Mutation $(-) n=18$} \\
\hline & 1 & 2 & 3 & 4 & 1 & 2 & 3 & 4 & 1 & 2 & 3 & 4 & 1 & 2 & 3 & 4 \\
\hline \multicolumn{17}{|l|}{ Haematological } \\
\hline Leucopoenia & 3 & 5 & 3 & 0 & 6 & 2 & 3 & 0 & 9 & 9 & 1 & 0 & 9 & 1 & 2 & 0 \\
\hline Neutropenia & 2 & 1 & 3 & 0 & 2 & 2 & 2 & 2 & 7 & 2 & 4 & 3 & 3 & 0 & 2 & 2 \\
\hline Febrile neutropenia & 0 & 1 & 0 & 0 & 2 & 1 & 0 & 0 & 4 & 1 & 0 & 0 & 2 & 0 & 0 & 0 \\
\hline Thrombocytopenia & 13 & 2 & 2 & 0 & 12 & 3 & 0 & 0 & 13 & 3 & 1 & 0 & 8 & 2 & 4 & 0 \\
\hline Anaemia & 7 & 12 & 3 & 0 & 3 & 12 & 3 & 0 & 5 & 14 & 5 & 0 & 3 & 12 & 2 & 0 \\
\hline \multicolumn{17}{|l|}{ Non-haematological } \\
\hline Nausea & 3 & 2 & 0 & 0 & 3 & 2 & 0 & 0 & 3 & 1 & 1 & 0 & 3 & 2 & 1 & 0 \\
\hline Vomiting & 3 & 3 & 1 & 0 & 2 & 3 & 0 & 0 & 5 & 0 & 1 & 0 & 2 & 4 & 0 & 0 \\
\hline Diarrhoea & 1 & 0 & 0 & 0 & 4 & 0 & 0 & 0 & 2 & 2 & 0 & 0 & 2 & 0 & 1 & 0 \\
\hline Elevated level of alanine & 8 & 2 & 2 & 0 & 7 & 0 & 0 & 1 & 13 & 4 & 2 & 0 & 6 & 1 & 2 & 0 \\
\hline \multicolumn{17}{|l|}{ aminotransferase } \\
\hline ILD-like syndrome & 0 & 0 & 0 & 0 & 0 & 0 & 0 & 0 & 0 & 0 & 1 & 0 & 0 & 0 & 0 & 0 \\
\hline Fever & 1 & 1 & 0 & 0 & 3 & 1 & 0 & 0 & 6 & 3 & 0 & 0 & 4 & 1 & 0 & 0 \\
\hline Rash & 0 & 1 & 0 & 0 & 0 & 1 & 0 & 0 & 12 & 6 & 1 & 0 & 5 & 1 & 2 & 1 \\
\hline Stomatitis/mucositis & 0 & 1 & 0 & 0 & 1 & 0 & 0 & 0 & 2 & 1 & 0 & 0 & 3 & 1 & 0 & 0 \\
\hline
\end{tabular}

Abbreviation: ILD, interstitial lung disease.

with gemcitabine plus erlotinib was 6.24 months [3]. The more favorable outcome is likely because of the higher rate of EGFR mutations in this study [19, 20], which enhanced treatment efficacy $[9,10,12,13]$. These results are encouraging, especially because all patients were already in the metastatic stage.

The median survival with gemcitabine alone in this trial (4.4 months) is lower than that found by Burris et al. (5.65 months) [2], Moore et al. (5.91 months) [3], Conroy et al. (FOLFIRINOX, 6.8 months) [4], and the Metastatic Pancreatic Adenocarcinoma Clinical Trial (MPACT, 6.6 months) [5]. In this trial, the disease control rate with gemcitabine alone $(25 \%)$ is also lower than that in the gemcitabine arms of the above trials (Burris et al., 44.4\%; Moore et al., 49.2\%; FOLFIRINOX, 50.9\%; MPACT, $33 \%$ ) [2-5]. Our results are similar to another study of patients of Chinese descent (median survival 4 months) [21]. Other than ethnic differences, advanced age (median age 70 years in this study vs. 61-64 years in other studies) and advanced stage (all stage IV) may also account for the relatively poor survival and lower disease control rate in the gemcitabine alone group in this study.

In NSCLC and breast cancer, the prevalence of EGFR mutations between Caucasian and Asian patients is different $[10,22]$. For NSCLC, there are variations between Chinese and other Asian populations [23, 24]. Lynch et al. did not detect any mutations in EGFR exons 19 and 21 in 40 pancreatic cancer primary tumors in Caucasian patients [9]. Tzeng et al. reported that 6/9 pancreatic cancer cell lines and 25/31 Caucasian pancreatic cancer patients had EGFR mutations, but all were silent mutations [20]. Oh et al. and Lee et al. showed a rate of $1.5-2.5 \%$ EGFR mutations in Korean pancreatic cancer cohorts [16, 25]. However, none of these patients was Chinese. Our study was conducted in Taiwan, where the majority of the population is of Chinese descent, and the pancreatic cancer EGFR mutation rate was $56 \%$. Improvement in OS for pancreatic cancer patients treated 
with erlotinib was apparent in our study because of the high proportion of cases with EGFR mutations compared to other studies. Currently, pancreatic cancer is the 7 th leading cause of cancer death among the Chinese, making $E G F R$ mutation detection an important issue in the treatment of pancreatic cancer [26].

The mutation sites in Chinese pancreatic cancer patients are also different from those reported in other populations $[16,25]$. The presence of EGFR-activating mutations in Chinese patients emphasizes ethnic, geographic, and environmental variations in pancreatic cancer mutation profiles that could result in different responses to erlotinib treatment. Similar to reports by Tzeng et al. and Lee et al., we found Q787Q to be the most common silent mutation $[16,20]$. In addition, we found four silent mutations that have not yet been reported, suggesting that EGFR silent mutations may vary among ethnicities.

The pattern of EGFR mutations varies among different solid tumors. In NSCLC, missense L858R and in-frame deletion E746_A750del constitute 78.2\% of mutations [27]. All mutations in exon 19 (7.3\%) were in-frame deletions (E746_A750del) in the squamous cell carcinoma of the head and neck [28], whereas two types of deletions, K745_E749del and E746_A750del, were found in exon 19 in cholangiocarcinomas [29]. Substitutions in exons 19 (E749K) and 20 (E762G and A767T) were predominant in $12 \%$ of colorectal carcinomas [30]. All of these mutations are different from those found in our work. Owing to these differences in the mutation profiles of $E G F R$ for pancreatic cancer, compared with other cancers, commercially available $E G F R$ mutation detection kits cannot replace direct sequencing [31].

Erlotinib-mediated disease control in EGFRmutated pancreatic cancer patients is modest, with many patients achieving stable disease $(85 \%)$. This is similar to the observations of Kwak et al. [19] who showed that both pancreatic cancer patients with EGFR mutations exhibited disease stabilization in response to therapy with erlotinib and capecitabine; however, this result does not fall within the same category of dramatic responses seen in NSCLC [9-14]. Further investigation is required to determine whether the site of primary cancer accounts for the disparate effects of TKI.

The gemcitabine plus erlotinib group had a higher disease control rate than the gemcitabine alone group for patients with an L778P mutation. Other mutation sites failed to demonstrate a significant difference in response and disease control rates. Because of their rarity, it is difficult to draw definitive conclusions regarding the true relationship between uncommon EGFR mutations and sensitivity to erlotinib. Further studies with larger patient numbers and functional analysis of the EGFR mutations are warranted to elucidate the impact of the individual mutation sites on the efficacy of erlotinib use.

There was no correlation between KRAS and EGFR mutations in our study, unlike the case in lung cancer, where these are generally mutually exclusive [32]. Furthermore, KRAS mutation status did not predict how patients would respond to erlotinib in our study, which is in line with the pivotal PA.3 study [17]. The small numbers of patients in our study $(5.7 \%)$ who had wildtype KRAS make comparisons difficult with data from other randomized trials.

Most adverse events associated with erlotinib plus gemcitabine treatment in this study were mild-tomoderate, consistent with previous studies [2-5]. Rash was more frequent with erlotinib plus gemcitabine than with gemcitabine alone, and we observed the association between rash and a better outcome that has been seen in other pancreatic cancer studies of EGFR inhibitors [3, 33, 34]. One patient in the gemcitabine plus erlotinib arm had ILD-like syndrome. Gemcitabine and EGFR TKIs are both known to cause ILD-like syndrome, and it is possible that there is a more-than-additive effect when these agents are combined [35]. The incidence seen in this study $(2.3 \%)$ is similar to that in other trials with combined gemcitabine and erlotinib [3].

FOLFIRINOX (a multidrug regimen of fluorouracil, leucovorin, irinotecan, and oxaliplatin) showed an increased median survival of 4.3 months but a worse safety profile compared to treatment with gemcitabine [4]. In another phase III multinational MPACT study, the combination of gemcitabine and nab-paclitaxel was shown to be superior to gemcitabine monotherapy, with a 1.8-month increase in median survival [5]. Combination therapy with gemcitabine plus erlotinib, gemcitabine plus nab-paclitaxel, or leucovorin, fluorouracil, irinotecan, and oxaliplatin is now considered first-line chemotherapy for metastatic pancreatic cancer.

Pancreatic cancer tends to be relatively resistant to chemotherapy and targeted therapy [6-8], and only a few regimens have proven efficacious [2-5, 36]. Our study first found that EGFR mutations are common in Chinese pancreatic cancer patients. In addition, this study showed that EGFR mutations can predict gemcitabine plus erlotinib treatment response and survival. However, this study did have some limitations. First, the sample size of 88 participants is relatively small. However, a strong association was still detected between EGFR mutations and the efficacy of erlotinib in pancreatic cancer. Second, all enrollees were Chinese, and these results might not apply to other ethnicities. These issues require further investigation in additional larger clinical studies.

In conclusion, this is the first study to show a significant association between EGFR mutations and survival benefit in metastatic pancreatic cancer patients receiving erlotinib combined with gemcitabine. Detection of EGFR mutations can be used to identify the subgroup of patients with pancreatic cancer in whom EGFR may be essential for tumor growth and who would thus benefit from treatment with erlotinib. 


\section{MATERIALS AND METHODS}

\section{Patient selection}

This study was a single-center, randomized, openlabel, prospective phase II trial. Inclusion criteria were metastatic pancreatic adenocarcinoma, presence of at least one measureable lesion, Eastern Cooperative Oncology Group (ECOG) performance status $\leq 2$, absolute neutrophil count $\geq 1,500 / \mathrm{mm}^{3}$, platelet count $\geq 100,000 /$ $\mathrm{mm}^{3}$, serum creatinine level $\leq 1.5 \times$ upper normal limit, aspartate aminotransferase or alanine aminotransferase level $\leq 5 \times$ upper normal limit, adequate samples for histological and mutational analyses, and absence of any other malignancy or serious medical or psychological illness that would preclude informed consent.

\section{Ethics statement}

Investigation has been conducted in accordance with the ethical standards and according to the Declaration of Helsinki and according to national and international guidelines and has been approved by the Institutional Review Board of Taipei Veterans General Hospital, conforms to CONSORT guidelines, and was registered as ClinicalTrial.gov NCT01608841.

\section{Randomization}

Patients were randomly allocated in a 1:1 ratio to receive either gemcitabine (Gemzar ${ }^{\circledR}$; Eli Lilly and Company, Indianapolis, IN, USA) plus erlotinib (Tarceva; OSI Pharmaceuticals, Farmingdale, NY, USA) or gemcitabine alone using a computerized random number function in Microsoft Excel (Microsoft, Redmond, WA, USA). A block randomization procedure was employed to ensure equal group allocation.

\section{Treatment}

Gemcitabine $1,000 \mathrm{mg} / \mathrm{m}^{2}$ was infused over 30 minutes on days $1,8,15,22,29,36$, and 43 followed by a 1 -week rest in cycle 1 , and on days 1,8 , and 15 in all subsequent 4-week cycles. Erlotinib was taken orally at $100 \mathrm{mg}$ once a day. Treatment continued until disease progression or intolerable toxicity.

\section{Pathology review, buffy coat, and DNA preparation}

Pathological specimens were obtained on initial diagnosis from sonography-guided (40 patients) or computed tomography-guided biopsy (15 patients), endoscopic biopsy (4 patients), open biopsy (9 patients), or pancreatectomy (20 patients). Tumor samples were obtained from primary pancreatic tumor $(n=44)$ or metastatic tissue $(n=44)$. All tissue sections were cut from formalin-fixed paraffin-embedded tumor blocks, and histological examination was performed by a pathologist (YCY) blinded to clinical information. All tumor regions were confirmed prior to DNA isolation. The buffy coat, representing the normal tissue and separated using Histopaque solution (Histopaque-1119; Sigma-Aldrich, St. Louis, MO, USA), was obtained from all patients at enrollment. DNA was extracted from sections and the buffy coat using the QIAamp DNA Mini kit (Qiagen, Hilden, Germany).

\section{Polymerase chain reaction (PCR) and sequencing of the EGFR gene}

Eight pairs of oligonucleotide primers were used to amplify exons $18-21$ of the EGFR gene using PCR as described previously (Supplementary Table 1) [9]. The PCR products were extracted and purified from a $1.5 \%$ agarose gel using the QIAquick gel extraction kit (Qiagen) and sequenced using the ABI PRISM BigDye Terminator Cycle Sequencing Ready Reaction kit and the ABI PRISM 3700 DNA Analyzer (PE Applied Biosystems, Foster City, CA, USA). PCR amplicon sequences were compared with the EGFR cDNA sequence obtained from Genbank (accession no. GI:22022643) by using Mutation Surveyor ${ }^{\text {TM }} 3.0$ (SoftGenetics, State College, PA, USA) and manual review. All sequence variants were verified by reverse sequencing.

\section{Ion torrent deep-amplicon sequencing}

To eliminate PCR artifacts and false positives, the GeneRead DNA FFPE kit was used to extract DNA. The DNA extracted from FFPE was used for multiplex PCR of a panel covering 18-21 exons in the EGFR gene. Fragment libraries were constructed using DNA fragmentation, barcode and adaptor ligation, and library amplification, according to the manufacturer's instructions, as stipulated in the Ion Xpress Plus Fragment Library Kit (Life Technologies, Grand Island, NY, USA). Size distribution of the DNA fragments was analyzed using the Agilent Bioanalyzer with the High Sensitivity Kit (Agilent, Santa Clara, CA, USA). Template preparation, emulsion PCR, and Ion Sphere Particle (ISP) enrichment was performed using the Ion PGM Template OT2 200 Kit (Life Technologies, Grand Island, NY, USA) according to the manufacturer's instructions. The ISPs were loaded onto a 314 chip and sequenced using an Ion PGM Sequencing 200 Kit v2 (Life Technologies, Grand Island, NY, USA). 


\section{Bioinformatics analysis}

After a successful sequencing reaction, the raw signal data were analyzed using Torrent Suite v4.0.2. The pipeline includes signaling processing, base calling, quality score assignment, adapter trimming, PCR duplicate removal, read alignment to human genome19 reference, mapping quality $\mathrm{QC}$, coverage analysis, and variant calling. After completion of the primary data analysis, lists of detected sequence variants (SNVs and INDELs) were compiled in the VCF (Variant call file) format. For downstream analysis, variants with a minimum coverage of 500 reads were selected.

\section{PCR and sequencing of the KRAS gene}

The cobas $^{\circledR}$ KRAS Mutation Test (Roche Diagnostics, Basel, Switzerland) was used according to the manufacturer's protocol [37]. Samples (50 ng of DNA) were aliquoted in 96-well plates, and negative and positive controls from the kit were added. Data were automatically processed by cobas ${ }^{\circledR}$ software.

\section{Evaluation of treatment response and safety}

Objective tumor response was evaluated every 8 weeks or earlier for patients with suspected progression according to the Response Evaluation Criteria in Solid Tumors (RECIST 1.0)[38]; responses were confirmed at least 28 days after the first documentation of a complete or partial response or stable disease. Images were blindly reviewed by a protocol-trained radiologist (RCL). Serial measurements of carbohydrate antigen 19-9 (CA19-9) were performed at baseline and every 4 weeks thereafter. Safety was monitored by assessing treatment-related adverse events and serious adverse events according to the National Cancer Institute Common Terminology Criteria for Adverse Events (v3.0).

\section{Statistical analysis}

A superiority test was conducted with the assumption that gemcitabine plus erlotinib would have a higher disease control rate [3], defined as the best overall response for complete or partial response or stable disease, than gemcitabine alone. The disease control rate was estimated as $30 \%$ in the gemcitabine alone group, and $60 \%$ in the gemcitabine plus erlotinib group [5, 39]. A sample size of 44 for each group was required for $80 \%$ power with 5\% error (two-tailed) and 5\% loss to followup [40]. Secondary outcome measures were response rate, progression-free survival (PFS), overall survival (OS), relation to $E G F R$ and $K R A S$ mutation status, and safety. PFS was defined as the time from random assignment to disease progression or death as a result of any cause. For patients alive without documented disease progression at the time of the analysis, PFS was censored at the time of the last tumor assessment. If no post-baseline assessment was performed, the date of random assignment plus 1 day was used as the censor date. OS was defined as the time from random assignment until death as a result of any cause, and patients alive at the time of the analysis were censored at the date of last contact. In the response rate analysis, patients without a post-baseline assessment were not assessed. PFS and OS were calculated using the Kaplan-Meier method and log-rank tests. The Student's t test or one-way analysis of variance was used to analyze continuous variables between groups. The association between skin rash, EGFR mutations, response rate, and disease control rate were compared using the Chi-square test or Fisher's exact test. Statistical analyses were performed using SPSS software (SPSS 21.0, SPSS Inc., Chicago, IL, USA). $P<0.05$ was considered statistically significant.

\section{ACKNOWLEDGMENTS}

The study was assisted in part by the Division of Experimental Surgery of the Department of Surgery, Taipei Veterans General Hospital to CPL. We thank Miss I-Hsuan Tsai at the Research and Development, TVGH Lihpao Lab of Cancer Genomic Medicine, Lihpao Life Science. Co., Ltd. for assisting with the next-generation sequencing.

\section{COMPETING INTERESTS}

We declare that we have no conflicts of interest.

\section{FUNDING}

The study was funded by Taipei Veterans General Hospital (V103C-201), the National Science Council (NSC 98-2314-B-075-029), the Research Foundation of Digestive Medicine, R.O.C. (10311), and the National Research Program for Biopharmaceutics of Taiwan (100CT202). The funders had no role in study design, data collection and analysis, decision to publish, or preparation of the manuscript.

\section{REFERENCES}

1. Siegel R, Ma J, Zou Z, Jemal A. Cancer statistics, 2014. CA Cancer J Clin 2014;64:9-29.

2. Burris HA, 3rd, Moore MJ, Andersen J, Green MR, Rothenberg ML, Modiano MR, et al. Improvements in survival and clinical benefit with gemcitabine as first-line therapy for patients with advanced pancreas cancer: a randomized trial. J Clin Oncol 1997;15:2403-2413. 
3. Moore MJ, Goldstein D, Hamm J, Figer A, Hecht JR, Gallinger S, et al. Erlotinib plus gemcitabine compared with gemcitabine alone in patients with advanced pancreatic cancer: a phase III trial of the National Cancer Institute of Canada Clinical Trials Group. J Clin Oncol 2007;25:19601966.

4. Conroy T, Desseigne F, Ychou M, Bouche O, Guimbaud $\mathrm{R}$, Becouarn Y, et al. FOLFIRINOX versus gemcitabine for metastatic pancreatic cancer. N Engl J Med 2011;364:18171825.

5. Von Hoff DD, Ervin T, Arena FP, Chiorean EG, Infante $\mathrm{J}$, Moore $\mathrm{M}$, et al. Increased survival in pancreatic cancer with nab-paclitaxel plus gemcitabine. N Engl J Med 2013;369:1691-1703.

6. Kindler HL, Ioka T, Richel DJ, Bennouna J, Letourneau R, Okusaka T, et al. Axitinib plus gemcitabine versus placebo plus gemcitabine in patients with advanced pancreatic adenocarcinoma: a double-blind randomised phase 3 study. Lancet Oncol 2011;12:256-262.

7. Philip PA, Benedetti J, Corless CL, Wong R, O’Reilly EM, Flynn PJ, et al. Phase III study comparing gemcitabine plus cetuximab versus gemcitabine in patients with advanced pancreatic adenocarcinoma: Southwest Oncology Group-directed intergroup trial S0205. J Clinic Oncol 2010;28:3605-3610.

8. Van Cutsem E, van de Velde H, Karasek P, Oettle H, Vervenne WL, Szawlowski A, et al. Phase III trial of gemcitabine plus tipifarnib compared with gemcitabine plus placebo in advanced pancreatic cancer. Journal of clinical oncology : official journal of the American Society of Clinical Oncology 2004;22:1430-1438.

9. Lynch TJ, Bell DW, Sordella R, Gurubhagavatula S, Okimoto RA, Brannigan BW, et al. Activating mutations in the epidermal growth factor receptor underlying responsiveness of non-small-cell lung cancer to gefitinib. N Engl J Med 2004;350:2129-2139.

10. Paez JG, Janne PA, Lee JC, Tracy S, Greulich H, Gabriel $\mathrm{S}$, et al. EGFR mutations in lung cancer: correlation with clinical response to gefitinib therapy. Science 2004;304:1497-1500.

11. Kosaka T, Yatabe Y, Endoh H, Kuwano H, Takahashi T, Mitsudomi T. Mutations of the epidermal growth factor receptor gene in lung cancer: biological and clinical implications. Cancer Res 2004;64:8919-8923.

12. Mu XL, Li LY, Zhang XT, Wang MZ, Feng RE, Cui $\mathrm{QC}$, et al. Gefitinib-sensitive mutations of the epidermal growth factor receptor tyrosine kinase domain in Chinese patients with non-small cell lung cancer. Clin Cancer Res 2005;11:4289-4294.

13. Pao W, Miller V, Zakowski M, Doherty J, Politi K, Sarkaria $\mathrm{I}$, et al. EGF receptor gene mutations are common in lung cancers from "never smokers" and are associated with sensitivity of tumors to gefitinib and erlotinib. Proc Natl Acad Sci U S A 2004;101:13306-13311.
14. Shepherd FA, Tsao MS. Unraveling the mystery of prognostic and predictive factors in epidermal growth factor receptor therapy. J Clin Oncol 2006;24:1219-1220.

15. Arteaga C. Targeting HER1/EGFR: a molecular approach to cancer therapy. Semin Oncol 2003;30:3-14.

16. Lee J, Jang KT, Ki CS, Lim T, Park YS, Lim HY, et al. Impact of epidermal growth factor receptor (EGFR) kinase mutations, EGFR gene amplifications, and KRAS mutations on survival of pancreatic adenocarcinoma. Cancer 2007;109:1561-1569.

17. da Cunha Santos G, Dhani N, Tu D, Chin K, Ludkovski $\mathrm{O}$, Kamel-Reid S, et al. Molecular predictors of outcome in a phase 3 study of gemcitabine and erlotinib therapy in patients with advanced pancreatic cancer: National Cancer Institute of Canada Clinical Trials Group Study PA.3. Cancer 2010;116:5599-5607.

18. Kim ST, Lim do H, Jang KT, Lim T, Lee J, Choi YL, et al. Impact of KRAS mutations on clinical outcomes in pancreatic cancer patients treated with first-line gemcitabine-based chemotherapy. Mol Cancer Ther 2011;10:1993-1999.

19. Kwak EL, Jankowski J, Thayer SP, Lauwers GY, Brannigan BW, Harris PL, et al. Epidermal growth factor receptor kinase domain mutations in esophageal and pancreatic adenocarcinomas. Clin Cancer Res 2006;12:4283-4287.

20. Tzeng CW, Frolov A, Frolova N, Jhala NC, Howard JH, Buchsbaum DJ, et al. Epidermal growth factor receptor (EGFR) is highly conserved in pancreatic cancer. Surgery 2007;141:464-469.

21. Meng Z, Garrett CR, Shen Y, Liu L, Yang P, Huo Y, et al. Prospective randomised evaluation of traditional Chinese medicine combined with chemotherapy: a randomised phase II study of wild toad extract plus gemcitabine in patients with advanced pancreatic adenocarcinomas. Br J Cancer 2012;107:411-416.

22. Lv N, Xie X, Ge Q, Lin S, Wang X, Kong Y, et al. Epidermal growth factor receptor in breast carcinoma: association between gene copy number and mutations. Diagn Pathol 2011;6:118.

23. Chou TY, Chiu CH, Li LH, Hsiao CY, Tzen CY, Chang KT, et al. Mutation in the tyrosine kinase domain of epidermal growth factor receptor is a predictive and prognostic factor for gefitinib treatment in patients with non-small cell lung cancer. Clin Cancer Res 2005;11:3750-3757.

24. Won YW, Han JY, Lee GK, Park SY, Lim KY, Yoon $\mathrm{KA}$, et al. Comparison of clinical outcome of patients with non-small-cell lung cancer harbouring epidermal growth factor receptor exon 19 or exon 21 mutations. J Clin Pathol 2011;64:947-952.

25. Oh DY, Lee KW, Lee KH, Sohn CH, Park YS, Zang DY, et al. A phase II trial of erlotinib in combination with gemcitabine and capecitabine in previously untreated metastatic/recurrent pancreatic cancer: combined analysis with translational research. Invest New Drugs 
2012;30:1164-1174.

26. Noormohammad SF, Mamlin BW, Biondich PG, McKown B, Kimaiyo SN, Were MC. Changing course to make clinical decision support work in an HIV clinic in Kenya. International Journal of Medical Informatics 2010;79:204210.

27. Yang CH, Yu CJ, Shih JY, Chang YC, Hu FC, Tsai MC, et al. Specific EGFR mutations predict treatment outcome of stage IIIB/IV patients with chemotherapy-naive non-smallcell lung cancer receiving first-line gefitinib monotherapy. Journal of clinical oncology : official journal of the American Society of Clinical Oncology 2008;26:27452753.

28. Lee JW, Soung YH, Kim SY, Nam HK, Park WS, Nam $\mathrm{SW}$, et al. Somatic mutations of EGFR gene in squamous cell carcinoma of the head and neck. Clin Cancer Res 2005;11:2879-2882.

29. Gwak GY, Yoon JH, Shin CM, Ahn YJ, Chung JK, Kim YA, et al. Detection of response-predicting mutations in the kinase domain of the epidermal growth factor receptor gene in cholangiocarcinomas. J Cancer Res Clin Oncol 2005;131:649-652.

30. Nagahara H, Mimori K, Ohta M, Utsunomiya T, Inoue H, Barnard GF, et al. Somatic mutations of epidermal growth factor receptor in colorectal carcinoma. Clin Cancer Res 2005;11:1368-1371.

31. Van Cutsem E, Li CP, Nowara E, Aprile G, Moore M, Federowicz I, et al. Dose escalation to rash for erlotinib plus gemcitabine for metastatic pancreatic cancer: the phase II RACHEL study. Br J Cancer 2014;111:2067-2075.

32. Pao W, Wang TY, Riely GJ, Miller VA, Pan Q, Ladanyi $\mathrm{M}$, et al. KRAS mutations and primary resistance of lung adenocarcinomas to gefitinib or erlotinib. PLoS Med 2005;2:e17.

33. Van Cutsem E, Vervenne WL, Bennouna J, Humblet Y, Gill $\mathrm{S}$, Van Laethem JL, et al. Phase III trial of bevacizumab in combination with gemcitabine and erlotinib in patients with metastatic pancreatic cancer. J Clin Oncol 2009;27:22312237.

34. Heinemann V, Vehling-Kaiser U, Waldschmidt D, Kettner E, Marten A, Winkelmann C, et al. Gemcitabine plus erlotinib followed by capecitabine versus capecitabine plus erlotinib followed by gemcitabine in advanced pancreatic cancer: final results of a randomised phase 3 trial of the 'Arbeitsgemeinschaft Internistische Onkologie' (AIOPK0104). Gut 2013;62:751-759.

35. Danson S, Blackhall F, Hulse P, Ranson M. Interstitial lung disease in lung cancer: separating disease progression from treatment effects. Drug Saf 2005;28:103-113.

36. Ueno H, Ioka T, Ikeda M, Ohkawa S, Yanagimoto H, Boku $\mathrm{N}$, et al. Randomized phase III study of gemcitabine plus S-1, S-1 alone, or gemcitabine alone in patients with locally advanced and metastatic pancreatic cancer in Japan and Taiwan: GEST study. J Clin Oncol 2013;31:1640-1648.
37. Lee S, Brophy VH, Cao J, Velez M, Hoeppner C, Soviero $\mathrm{S}$, et al. Analytical performance of a PCR assay for the detection of KRAS mutations (codons 12/13 and 61) in formalin-fixed paraffin-embedded tissue samples of colorectal carcinoma. Virchows Arch 2012;460:141-149.

38. Therasse P, Arbuck SG, Eisenhauer EA, Wanders J, Kaplan RS, Rubinstein L, et al. New guidelines to evaluate the response to treatment in solid tumors. European Organization for Research and Treatment of Cancer, National Cancer Institute of the United States, National Cancer Institute of Canada. J Natl Cancer Inst 2000;92:205216.

39. Chao Y, Wu CY, Wang JP, Lee RC, Lee WP, Li CP. A randomized controlled trial of gemcitabine plus cisplatin versus gemcitabine alone in the treatment of metastatic pancreatic cancer. Cancer Chemother Pharmacol 2013;72:637-642.

40. http://www.stat.ubc.ca/ rollin/stats/ssize/b2.html. 